\title{
Atom gravimeters and gravitational redshift
}

\author{
Arising from: H. Müller, A. Peters \& S. Chu Nature 463, 926-929 (2010).
}

In ref. 1 the authors present a re-interpretation of atom interferometry experiments published a decade ago $^{2}$. They now consider the atom interferometry experiments ${ }^{2}$ as a measurement of the gravitational redshift on the quantum clock operating at the Compton frequency $\omega_{\mathrm{C}}=m c^{2} / \hbar \approx 2 \pi \times 3.0 \times 10^{25} \mathrm{~Hz}$, where $m$ is the caesium (Cs) atom rest mass. They then argue that this redshift measurement compares favourably with existing ${ }^{3}$ as well as projected ${ }^{4}$ clock tests. Here we show that this interpretation is incorrect.

Since their publication, atom interferometry experiments of this type have been analysed as measuring the acceleration of free fall of Cs atoms in the interferometer, leading to a test of the universality of free fall (UFF) through a comparison with measurements using a freely falling corner cube. Although it remains less sensitive than tests using macroscopic test masses of different composition ${ }^{5,6}$ (relative precision $7 \times 10^{-9}$ versus $2 \times 10^{-13}$ ), this UFF test is very important because it is the most sensitive test that compares the free fall of quantum bodies with that of classical test masses.

But do such experiments measure the gravitational redshift? We first emphasize that the atom interferometer used in ref. 2 is an accelerometer (or gravimeter). The associated signal is a phase shift $\Delta \varphi=\mathbf{k} \cdot \mathbf{g} T^{2}$, where $\mathbf{k}$ is the effective wavevector transferred by lasers, $\mathbf{g}$ is the local acceleration of gravity and $T$ is the interrogation time. It measures the acceleration of freely falling atoms, as defined with respect to the experimental platform that holds the optical and laser elements. With $\mathbf{k}$ and $T$ known from auxiliary measurements, one deduces the component of $\mathbf{g}$ along the direction of $\mathbf{k}$. If the whole instrument was put into a freely falling laboratory, the phase shift $\Delta \varphi$ would vanish.

The situation is completely different for instruments used for testing the universality of clock rates (UCR). An atomic clock delivers a periodic electromagnetic signal the frequency of which is actively controlled to remain tuned to an atomic transition. The clock frequency is sensitive to the gravitational potential $U$ and not to the local gravity field $\mathbf{g}=\nabla U$. UCR tests are then performed by comparing clocks through the exchange of electromagnetic signals; if the clocks are at different gravitational potentials, this contributes to the relative frequency difference by $\Delta v / v=\Delta U / c^{2}$.

We show now that ref. 1 does not measure the gravitational redshift. This is clearly seen when evaluating the phase shift $\Delta \varphi$ using a Lagrangian formulation as employed in ref. 1; the Lagrangian may for example have the form considered in equation (8) of ref. 1 . It can then be proved that the clock contribution $\Delta \varphi_{\text {free }}$ discussed in ref. 1 -that is, the difference of Compton phases along the two paths-is exactly zero for a closed total path (equation (81) in ref. 7), or exactly cancelled by a second contribution associated with the splitting of the endpoints of these paths ${ }^{8}$. The final phase shift $\Delta \varphi$ then arises entirely from a third contribution describing the interaction of light with atoms in the beam-splitting process. This explains why the phase shift takes the already discussed expression $\mathbf{k} \cdot \mathbf{g} T^{2}$, which does not depend on the Compton frequency $\omega_{\mathrm{C}}$.

The key point in this cancellation is a consistent calculation of the two paths in the atom interferometer as well as of the phases along these paths, both derived from the same Lagrangian by using the principle of least action in a standard way. When this is done in the case of ref. 1 , we find that their equation (8) gives $\Delta \varphi_{\text {free }}=0$, irrespective of the value of the redshift violation parameter $\beta$. If one doubts the validity of this principle of least action, the significance and sensitivity of the test of ref. 1 remains to be evaluated ${ }^{9}$.

To summarize, the experiment discussed in refs 1 and 2 is an interesting test of UFF but not a measurement of the gravitational redshift or a test of UCR. Both kinds of tests (UFF and UCR) have their own merit, because they check in different ways whether all types of mass-energy are universally coupled to gravitation ${ }^{10}$.

Peter Wolf ${ }^{1}$, Luc Blanchet ${ }^{2}$, Christian J. Bordé ${ }^{1}$, Serge Reynaud ${ }^{3}$, Christophe Salomon ${ }^{4} \&$ Claude Cohen-Tannoudji ${ }^{4}$

${ }^{1}$ Systèmes de Références Temps Espace, CNRS UMR 8630, Laboratoire National de Métrologie et d'Essais, Université Pierre et Marie Curie, Observatoire de Paris, 61 avenue de I'Observatoire, 75014 Paris, France. e-mail: peter.wolf@obspm.fr

${ }^{2}$ GRECO, Institut d'Astrophysique de Paris, CNRS UMR 7095, UPMC,

98 bis boulevard Arago, 75014 Paris, France.

${ }^{3}$ Laboratoire Kastler Brossel, CNRS UMR 8552, Ecole Normale

Supérieure, UPMC, Campus Jussieu, 75252 Paris, France.

${ }^{4}$ Laboratoire Kastler Brossel and Collège de France, CNRS UMR 8552,

ENS, UPMC, 24 rue Lhomond, 75231 Paris, France.

Received 23 April; accepted 23 June 2010.

1. Müller, H., Peters, A. \& Chu, S. A precision measurement of the gravitational redshift by the interference of matter waves. Nature 463, 926-929 (2010).

2. Peters, A., Chung, K. Y. \& Chu, S. A measurement of gravitational acceleration by dropping atoms. Nature 400, 849-852 (1999).

3. Vessot, R. F. C. et al. Test of relativistic gravitation with a space-borne hydrogen maser. Phys. Rev. Lett. 45, 2081-2084 (1980).

4. Cacciapuoti, L. \& Salomon, C. Space clocks and fundamental tests: the ACES experiment. Eur. Phys. J. Spec. Top. 127, 57-68 (2009).

5. Williams, J. G., Turyshev, S. G. \& Boggs, D. H. Progress in lunar laser ranging tests of relativistic gravity. Phys. Rev. Lett. 93, 261101 (2004).

6. Schlamminger, $\mathrm{S}$. et al. Test of the equivalence principle using a rotating torsion balance. Phys. Rev. Lett. 100, 041101 (2008).

7. Storey, P. \& Cohen-Tannoudji, C. The Feynman path integral approach to atomic interferometry. A tutorial. J. Phys. II 4, 1999-2027 (1994).

8. Bordé, C. J. 5D optics for atomic clocks and gravito-inertial sensors. Eur. Phys. J. Spec. Top. 163, 315 (2008).

9. Will, C. M. Theory and Experiments in Gravitational Physics Ch. 2 (Cambridge University Press, 2000).

10. Damour, T. Experimental tests of gravitational theory. In The Review of Particle Physics (ed. Amsler, C. et al.) Phys. Lett. B667, 1 (2008); 〈http://pdg.lbl.gov/〉.

Competing financial interests: declared none.

doi:10.1038/nature09340 


\section{Müller, Peters \& Chu reply}

Replying to: P. Wolf, L. Blanchet, C. J. Bordé, S. Reynaud, C. Salomon \& C. Cohen-Tannoudji Nature doi:10.1038/nature09340 (2010)

We stand by our result ${ }^{1}$. The Comment ${ }^{2}$ revisits an interesting issue that has been known for decades ${ }^{3-5}$. Because it applies to all experiments, classical redshift tests ${ }^{6,7}$ and atom interferometry redshift tests are equivalent for all aspects in question.

We first note that no experiment is sensitive to the absolute value of a potential $U$. When two similar clocks at rest in the laboratory frame are compared in a classical redshift test, their frequency difference $\Delta v / v=\Delta U / c^{2}$ is given by $\Delta U=\mathbf{g h}+\mathcal{O}\left(h^{2}\right)$, where $\mathbf{g}=\nabla U$ is the gravitational acceleration in the laboratory frame, $\mathbf{h}$ is the clock's separation, $c$ is the velocity of light, and $\mathcal{O}\left(h^{2}\right)$ indicates terms of order $h^{2}$ and higher. Therefore, classical redshift tests are sensitive to $\mathbf{g}$, not to the absolute value of $U$, just like atom interferometry redshift tests. For classical redshift tests, as for any experiment, all effects of conventional gravity, including the redshift, are eliminated locally inside a freely falling laboratory ${ }^{8}$. If the clock's trajectory is tracked from a fixed ground station, the complete experiment is not in free fall. Also, the redshift is $\Delta U / c^{2}$, independent of whether the clocks are compared by exchange of electromagnetic signals or clock transport ${ }^{8}$.

The atom interferometry signal $\varphi=\varphi_{\mathrm{r}}+\varphi_{\mathrm{t}}+\varphi_{\mathrm{i}}$ is the sum of contributions of the redshift $\varphi_{\mathrm{r}}=\mathbf{k g} T^{2}=\left(m c^{2} / \hbar\right) \int\left(\Delta U / c^{2}\right) \mathrm{d} t$ to the Compton frequency $\omega_{\mathrm{C}}=m c^{2} / \hbar$ (where $\mathbf{k}$ is the wavevector, $T$ the pulse separation time ${ }^{1}, m$ the rest mass of the atom, and $\hbar$ the reduced Planck constant), time dilation $\varphi_{\mathrm{t}}$, and the laser-atom interaction $\varphi_{\mathrm{i}}$. As is common in classical redshift tests, the time dilation $\varphi_{\mathrm{t}}$ due to the clocks' motion is compensated for, so that a measurement of $\varphi_{\mathrm{r}}$ is obtained. This happens automatically, because $\varphi_{\mathrm{t}}+\varphi_{\mathrm{i}}=0$. By interfering the atomic matter waves from the two paths, we obtain $\varphi$, which contains their proper time difference? ${ }^{9}$. To compare to Einstein's prediction, we measure $g$ by tracking a falling corner cube's position (apart from its proper time) by interfering light reflected off the corner cube.

As has been pointed out ${ }^{4,8,10}$, the Einstein equivalence principle does not follow from tests of the universality of free fall (UFF) without redshift measurements. The Comment ${ }^{2}$, however, argues that we must use the Lagrangian equation (8) to derive the paths as well as the phases. It thus uses a case where measuring the redshift and testing UFF are equivalent and so the Einstein equivalence principle does follow from tests of UFF alone ${ }^{1}$. In this case, the Comment is correct that $\Delta \varphi_{\text {free }}=\varphi_{\mathrm{r}}+\varphi_{\mathrm{t}}=0$, and thus atom interferometers measure the acceleration of free fall. Simultaneously, we are correct that $\varphi_{\mathrm{t}}+\varphi_{\mathrm{i}}=0$, showing that they measure the redshift. Using this approach, however, precludes redshift anomalies without corresponding UFF violation. Any anomaly would cancel upon comparing measured and predicted redshifts, because the anomaly would also be contained in $g$. This has been shown explicitly for classical redshift tests ${ }^{5}$, and atom interferometers are no different in this respect.

The more general-and more interesting — scenario is that redshift and UFF violations can exist independently; explicit theoretical models for this have been proposed using, for example, non-minimal coupling $^{11,12}$ or gravitoscalar and gravitovector fields ${ }^{13}$. Then $\Delta \varphi_{\text {free }}=\varphi_{\mathrm{r}}+\varphi_{\mathrm{t}}$ is no longer zero, but our $\varphi_{\mathrm{t}}+\varphi_{\mathrm{i}}=0$ still holds ${ }^{1}$, even for arbitrary simultaneous violations of UFF. Given that a complete theory exhibiting redshift anomalies while preserving UFF is not yet known, it would be premature to use a detailed model for such physics. Our analysis consequently allows (but does not require) the free-fall acceleration of the atoms $g^{\prime}$ and the redshift to be independent. Similar assumptions have been applied to all previous ${ }^{5}$ and planned $^{6}$ redshift experiments.

Atom interferometry redshift tests and classical redshift tests are both valid measurements of the gravitational redshift. Both remain important, because they test the Einstein equivalence principle over complementary parameters like elevation $(1,000 \mathrm{~km}$ versus $1 \mathrm{~mm})$, clock frequencies $\left(10^{9} \mathrm{~Hz}\right.$ versus $10^{25} \mathrm{~Hz}$ ), clock mechanisms (mostly hyperfine interactions versus mostly strong interactions), and methods (radio link versus clock transport). Considering the wide range of scenarios for physics beyond the standard model, it is important to probe the redshift on all experimentally accessible scales.

Holger Müller, ${ }^{1,2}$ Achim Peters ${ }^{3}$ \& Steven Chu ${ }^{1,2,4}$

${ }^{1}$ Department of Physics, 151 Le Conte Hall MS 7300, University of

California, Berkeley, California 94720, USA.

e-mail: hm@berkeley.edu

${ }^{2}$ Lawrence Berkeley National Laboratory, One Cyclotron Road, Berkeley, California 94720, USA.

${ }^{3}$ Institut für Physik, Humboldt-Universität zu Berlin, Hausvogteiplatz 5-7, Berlin 10117, Germany.

${ }^{4}$ US Department of Energy, 1000 Independence Avenue SW,

Washington, District of Columbia 20585, USA.

1. Müller, H., Peters, A. \& Chu, S. A precision measurement of the gravitational redshift by the interference of matter waves. Nature 463, 926-929 (2010).

2. Wolf, P. et al. Atom gravimeters and gravitational redshift. Nature doi: 10.1038/ nature09340 (2010).

3. Schiff, L. On experimental tests of the general theory of relativity. Am. J. Phys. 28, 340-343 (1960).

4. Dicke, R. H. Eötvös experiment and the gravitational red shift. Am. J. Phys. 28, 344-347 (1960).

5. Nordtvedt, K. Quantitative relationship between clock gravitational "red-shift" violations and nonuniversality of free-fall rates in nonmetric theories of gravity. Phys. Rev. D 11, 245-247 (1975).

6. Vessot, R. F. C. et al. Test of relativistic gravitation with a space-borne hydrogen maser. Phys. Rev. Lett. 45, 2081-2084 (1980).

7. Cacciapuoti, L. \& Salomon, C. Space clocks and fundamental tests: the ACES experiment. Eur. Phys. J. Spec. Top. 127, 57-68 (2009).

8. Will, C. M. Theory and Experiment in Gravitational Physics Ch. 2 (Cambridge University Press, 1993).

9. Bordé, C. J. 5D optics for atomic clocks and gravito-inertial sensors. Eur. Phys. J. Spec. Top. 163, 315-332 (2008).

10. Will, C. M. The confrontation between general relativity and experiment. Living Rev. Relativity 9, 3 (2006); 〈http://relativity.livingreviews.org/Articles/Irr-2006-3/〉.

11. Coley, A. Schiff's conjecture on gravitation. Phys. Rev. Lett. 49, 853-855 (1982).

12. Ni, W.-T. Equivalence principles and electromagnetism. Phys. Rev. Lett. 38, 301-304 (1977).

13. Nieto, M. M. \& Goldmann, T. The arguments against "antigravity" and the gravitational acceleration of antimatter. Phys. Rep. 205, 221-281 (1991).

Author Contributions All authors have contributed substantially to this work.

Competing financial interests: declared none.

doi:10.1038/nature09341 